Report

\title{
Diet and risk for breast cancer recurrence and survival
}

\author{
Gordon A. Saxe ${ }^{1}$, Cheryl L. Rock ${ }^{2}$, Max S. Wicha ${ }^{3}$, and David Schottenfeld ${ }^{4}$ \\ ${ }^{1}$ Department of Medicine, Berkshire Medical Center, and University of Massachusetts Medical School, USA; \\ ${ }^{2}$ Department of Family and Preventive Medicine, Cancer Prevention and Control Program, University of California, \\ San Diego, USA; ${ }^{3}$ Department of Medicine, School of Medicine, University of Michigan, USA; ${ }^{4}$ Department of \\ Epidemiology, School of Public Health, University of Michigan, USA
}

Key words: breast cancer, diet, recurrence, survival

\begin{abstract}
Summary
Dietary factors may influence the risk for breast cancer and also the prognosis following diagnosis and treatment. The aim of this study was to assess whether self-reported prediagnosis diet or other patient factors associated with breast cancer incidence were predictive of recurrence and survival. Patients $(n=149)$ diagnosed with primary breast cancer between 1989 and 1991 were followed for five or more years. Total energy (hazard ratio $(\mathrm{HR})=1.58$, $95 \%$, confidence interval $(\mathrm{CI})=1.05,2.38)$ as well as total $(\mathrm{HR}=1.46,95 \% \mathrm{CI}=1.05,2.01)$, saturated $(\mathrm{HR}=$ $1.79,95 \% \mathrm{CI}=1.05,3.04)$, and monounsaturated $(\mathrm{HR}=1.65,95 \% \mathrm{CI}=1.09,2.49)$ fat intakes were associated with increased risk, and energy-adjusted bread and cereal consumption ( $\mathrm{HR}=0.55,95 \% \mathrm{CI}=0.33,0.93)$ with decreased risk of recurrence. Both total energy $(\mathrm{HR}=1.58,95 \% \mathrm{CI}=1.03,2.43)$ and polyunsaturated fat $(\mathrm{HR}=1.84,95 \% \mathrm{CI}=1.09,3.13)$ intakes were associated with an increased risk of death. All associations between dietary fat and recurrence and survival attenuated following energy adjustment. Oral contraceptive use $(\mathrm{HR}=1.28,95 \% \mathrm{CI}=1.03,1.60)$, lymph node positive status $(\mathrm{HR}=2.36,95 \% \mathrm{CI}=1.01,5.49)$, and tumor stage $(\mathrm{HR}=2.22,95 \% \mathrm{CI}=1.02,4.81)$ were associated with increased risk of recurrence. Tumor stage $(\mathrm{HR}=$ $4.96,95 \% \mathrm{CI}=1.86,13.23)$, lymph node positive status $(\mathrm{HR}=3.31,95 \% \mathrm{CI}=1.38,7.95)$, and estrogen receptor negative status $(\mathrm{HR}=2.46,95 \% \mathrm{CI}=1.02,5.94)$ were associated with increased risk, and arm muscle circumference $(\mathrm{HR}=0.27,95 \% \mathrm{CI}=0.09,0.86)$ and mammographic utilization $(\mathrm{HR}=0.77,95 \% \mathrm{CI}=$ $0.61,0.98)$ with decreased risk of death. Higher levels of energy, fat intakes, and selected patient characteristics (particularly disease stage and anthropometric indicators of adiposity) appear to increase risk of recurrence and/or shortened survival following the diagnosis of breast cancer.
\end{abstract}

\section{Introduction}

Ecological and analytical epidemiologic investigations and laboratory studies have suggested that dietary factors may influence the genesis and incidence of breast cancer. More recently, the question has been raised whether diet or nutritional factors, such as body weight, may continue to influence the natural history of this disease after diagnosis and treatment, thereby modifying risks of recurrence and death.

Several studies have associated decreased survival with prediagnosis fat intake, adjusted [1-3] or unadjusted [4-6] for energy intake, whereas others [7-9] failed to detect any association with dietary fat. Some of these studies [1-3] found a harmful effect of fat in- take, independent of energy, whereas others, in which fat estimates were not energy-adjusted, were unable to disentangle the effects of these closely-related factors. Other, but not all, studies have also found protective effects of intake of vegetables and fruits and associated micronutrients, vitamin $\mathrm{C}$ and carotenoids [1, 3, 10]. Obesity may decrease survival $[4,9,11-15]$, although it is not known whether this reflects excess intake of energy and fat or influences risk for independent reasons.

The purpose of this study was to examine the effect on prognosis of both dietary intakes and other patient factors, such as reproductive events, body mass, and tumor characteristics, considering the endpoint of disease recurrence in addition to survival, in a group 
of women with primary breast cancer who had been identified and examined upon diagnosis of the disease. We also examined whether the effects of dietary factors varied according to menstrual status, body mass, and disease stage, which are critical variables with the potential to confound or modify diet-disease outcome relationships. This study also builds on an earlier study [16], in which we explored relationships between diet and prognostic tumor characteristics, by examining whether dietary factors are related to rates of recurrence and survival of treated patients.

\section{Patients and methods}

\section{Patients}

Subjects consisted of 149 primary breast cancer patients who had been seen for the first time during an 18-month period beginning in August, 1989, at the Medical Center, University of Michigan. A patient was excluded if (1) the diagnosis of breast cancer was not biopsy-confirmed, (2) more than one month had elapsed since her primary surgery was performed, (3) the diagnosis at examination was of recurrent breast cancer, (4) the dietary questionnaire was rendered unusable by numerous omissions, or (5) the patient was unable to be interviewed for the study. Procedures for this study were approved by the Institutional Review Board of the School of Medicine, University of Michigan.

\section{Collection of dietary and other patient data}

Study data were obtained early in the course of the patient's initial care. An interview was conducted with each suspected or newly diagnosed breast cancer patient as close to the time of her breast cancer diagnosis as possible (usually her initial clinic visit or, failing that, the first post-operative visit). At the time of this interview, dietary and anthropometric assessments were performed, and the patient was queried about her menstrual history and use of oral contraceptives (OC) and hormone replacement therapy (HRT). She was also asked to complete a questionnaire regarding demographic background and reproductive history.

Anthropometric measurements, including height, weight, triceps skinfold thickness (TSF), and midarm circumference (MAC) were obtained by standard procedures [17]. Calculated indices included body mass index $(\mathrm{BMI})=$ weight $(\mathrm{kg}) /$ height $\left(\mathrm{m}^{2}\right)$ and arm muscle circumference $(\mathrm{AMC})(\mathrm{cm})=\mathrm{MAC}-\pi \times \mathrm{TSF}$. Body mass index was corrected for arm muscle circumference $\left(\mathrm{kg} / \mathrm{m}^{2} / \mathrm{cm}\right)=$ BMI/AMC [17], because sole reliance on weight or BMI can lead to misclassification of some individuals as obese. Dividing BMI by arm muscle circumference (BMI/AMC) provides an index of adiposity which may be more accurate than BMI alone because muscularity is thus considered in the evaluation of the effect of body mass. This approach is analogous to and modeled on the nutrient density method of energy adjustment [18-20].

Tumor characteristics, including stage, lymph node status, and estrogen receptor (ER) levels and status, were obtained by review of hospital medical records or, when necessary, by contacting outside institutions for information which was missing from medical records. Staging was based on the American Joint Commission on Cancer system [21]. Biopsy specimens obtained to ascertain diagnosis, either at the University of Michigan Hospital or outside hospitals, were used to stage the primary tumor and were assayed by the Department of Pathology Ligand Laboratory using the biochemical, dextran-coated charcoal method to determine ER levels. The cut-point used to dichotomize ER status as positive or negative was $10 \mathrm{fmol}$ receptor protein $/ \mathrm{mg}$ cytosol. When insufficient biopsy tissue was available for this method, a determination of receptor status was made using immunohistochemical staining. Lymph node status was based on the pathological findings of axillary node dissections performed at the time of primary surgery. The presence or absence of metastatic disease was based on the findings of a metastatic workup which included liver function tests and, when appropriate, bone or computed tomography scans and biopsy of suspicious lesions.

\section{Dietary assessment}

Nutrient intakes were examined using a National Cancer Institute food frequency questionnaire (FFQ) [22]. The FFQ was designed to assess a subject's usual diet and to provide information on a variety of nutrients, foods, and food groups. Approximately 100 food items were selected for inclusion in the questionnaire on the basis of their estimated contributions to the intake of a variety of nutrients, particularly fat and vitamin A, by the US population. The FFQ was semi-quantified, so that a standard portion size for each of the food items was presented, and the subjects were asked to indicate both the frequency of their consumption of the item and whether their usual intake was larger, the same, or smaller than an indicated standard amount.

The FFQ was administered at the time of a patient's first clinic visit, with a focus on the typical diet during the year prior to diagnosis. Each patient was taught how to complete the form and was asked 
to complete representative items in the presence of an interviewer. Completed FFQs were collected by the interviewer and checked for completeness, accuracy, phase shifts, and unusual responses. Patients with problematic FFQ responses were queried for correct information and when necessary, entire questionnaires were interviewer-administered.

Traditional analytic methods used to assess food content involved quantifying the carotenoids believed to be potential precursors of vitamin A, with results tabulated as vitamin A values in international units or retinol equivalents [23]. With the nutrient content database and software employed in this study, vitamin A intake was quantified as total vitamin A and its two components, preformed vitamin A (retinol) and 'carotene' (assumed to reflect the major vitamin A precursors). A more recently released database, with figures derived from modern HPLC methods, permits increased accuracy in the assessment of carotenoids in the diet [24, 25]. However, the correlation between the intakes based on the early estimates and the new food composition values has been found to be very high [26], so that use of these data in analysis is expected to approximate the relative carotenoid intakes across the range consumed within a target group.

\section{Recurrence and mortality data collection}

Access was granted to the database of the University of Michigan Tumor Registry. The Registry tracks all breast cancer patients who have been treated at the University Hospital with annual updates of their recurrence and survival status. Each of the breast cancer patients who had completed a FFQ at diagnosis was searched using this system, yielding 149 cases who, because they received their primary care at the University Hospital, had been actively followed. In cases where the patient had suffered a recurrence, the date of the diagnosis was noted, along with the type of recurrence (local, regional, or distant). Similarly, for patients who died, the date of death, as well as whether the death was breast-cancer related, was noted. All recurrences (local, regional, or distant) were analyzed together, as were all deaths (breast-cancer related and unrelated).

\section{Analysis}

All data were entered into a computer-based file and analyzed using SPSS for Windows (version 6.1.4, 1996, SPSS, Chicago, IL). Descriptive statistics (means, standard deviations, minima, maxima, and frequency distributions) were calculated for all study variables and were examined to determine whether assump- tions of normal distribution were met. Variables not normally distributed were log-transformed to achieve normality prior to analysis. Correlations among the independent variables were examined using Pearson product-moment correlations. A series of Cox proportional hazards analyses [27] was then conducted to model each study variable in relation to both recurrence and survival within the population as a whole. Variable increments used in the proportional hazards analyses were selected on the basis of distribution of the data collected. Dietary variables were examined both before and after adjusting for energy. Energy adjustment was carried out using the nutrient density method [18]. Analyses by others $[19,20]$ indicate that this method provides the greatest power to detect associations and estimates of risk which are the most stable and closest to the null.

In a second set of Cox proportional hazards analyses, energy-adjusted dietary variables were then examined in relation to both recurrence and survival within menstrual status (pre- vs. postmenopausal), BMI ( $\leq$ $27.0 \mathrm{vs}$. $>27.0 \mathrm{~kg} / \mathrm{m}^{2}$ ), and disease stage (Stages 0 and I vs. II-IV) strata. A BMI cut-point of $27.0 \mathrm{~kg} / \mathrm{m}^{2}$ was selected because this is the level at which adverse health effects from overweight are believed to begin to occur [28]. Finally, a series of multivariate Cox analyses were performed, using a stepwise selection procedure, to arrive at the strongest dietary (dietary variables only) and overall (both dietary and non-dietary variables) models of both recurrence and survival. For descriptive purposes, means \pm SD are reported.

\section{Results}

Table 1 provides a description of the study population in terms of their demographic, reproductive, anthropometric, dietary, and disease-related characteristics. Bivariate Pearson correlations between selected dietary and patient variables revealed several significant associations. Energy intake was strongly correlated with other dietary variables, including total dietary fat $(r=$ $0.90 ; P<0.001)$, carotene $(r=0.38 ; P<0.001)$, and bread and cereals $(r=0.36 ; P<0.001)$, but not with BMI. Carotene intake was strongly correlated with green and yellow vegetables $(r=0.84 ; P<0.001)$ and total dietary fat $(r=0.27 ; P<0.001)$ intakes, but not with intake of breads and cereals. BMI was strongly correlated with weight $(r=0.95 ; P<0.001)$, triceps skinfold thickness $(r=0.71 ; P<0.001)$, and arm muscle circumference $(r=0.56 ; P<0.001)$. 
Table 1. Summary statistics for study variables $(n=149)$

\begin{tabular}{|c|c|c|c|}
\hline Variable & & $\begin{array}{l}\text { Mean } \pm \\
\text { standard deviation }\end{array}$ & Range \\
\hline \multicolumn{4}{|l|}{ A. Patient characteristics } \\
\hline Age (yr) & & $57.8 \pm 13.4$ & $26-95$ \\
\hline Age at menarche (yr) & & $13.2 \pm 3.1$ & $10-16$ \\
\hline Age at menopause (yr) & & $46.6 \pm 7.6$ & $25-59$ \\
\hline Parity & & $2.7 \pm 1.5$ & $0-7$ \\
\hline Age at first birth (yr) & & $24.7 \pm 5.8$ & $15-44$ \\
\hline Number of children breastfed & & $0.9 \pm 1.4$ & $0-6$ \\
\hline Body weight (kg) & & $70.0 \pm 16.3$ & $45.5-123.6$ \\
\hline Height $(\mathrm{cm})$ & & $160.8 \pm 11.7$ & $113.0-179.1$ \\
\hline Body mass index $\left(\mathrm{kg} / \mathrm{m}^{2}\right)$ & & $26.7 \pm 5.9$ & $15.7-48.9$ \\
\hline Arm muscle circumference $(\mathrm{cm})$ & & $20.3 \pm 3.4$ & $2.1-29.2$ \\
\hline Oral contraceptive use (mon) & & $24.4 \pm 47.5$ & $0-264$ \\
\hline Hormone replacement use (mon) & & $22.9 \pm 52.2$ & $0-324$ \\
\hline Number of mammograms in past 5 years & & $2.8 \pm 3.9$ & $0-14$ \\
\hline \multicolumn{4}{|l|}{ B. Dietary variables } \\
\hline Total energy $(\mathrm{kcal} / \mathrm{d})$ & & $1671.4 \pm 755.2$ & $451.7-5067.9$ \\
\hline Fat, energy ( $\%$ of kcal) & & $32.2 \pm 7.0$ & $11.3-50.2$ \\
\hline Protein, energy (\% of kcal) & & $16.8 \pm 2.9$ & $10.2-25.6$ \\
\hline Carbohydrate, energy (\% of kcal) & & $49.1 \pm 8.4$ & $31.2-74.2$ \\
\hline Total fat $(\mathrm{g} / \mathrm{d})$ & & $60.4 \pm 30.4$ & $10.7-184.7$ \\
\hline Saturated fat $(\mathrm{g} / \mathrm{d})$ & & $22.0 \pm 11.9$ & $3.8-78.9$ \\
\hline Monounsaturated fat (g/d) & & $22.0 \pm 11.7$ & $2.9-70.3$ \\
\hline Polyunsaturated fat $(\mathrm{g} / \mathrm{d})$ & & $10.9 \pm 6.2$ & $1.3-32.2$ \\
\hline Fiber $(\mathrm{g} / \mathrm{d})$ & & $15.5 \pm 9.6$ & $2.9-58.2$ \\
\hline Vitamin C $(\mathrm{mg} / \mathrm{d})$ & & $161.2 \pm 114.5$ & $21.0-636.9$ \\
\hline Carotene $(\mu \mathrm{g} / \mathrm{d})$ & & $3543.9 \pm 3395.1$ & $334.3-26778.9$ \\
\hline Retinol $(\mu \mathrm{g} / \mathrm{d})$ & & $874.2 \pm 747.7$ & $80.0-5089.0$ \\
\hline Fruit (servings/week) & & $16.7 \pm 13.5$ & $0.6-85.5$ \\
\hline Green and yellow vegetables (servings/week) & & $4.1 \pm 3.7$ & $0.1-23.0$ \\
\hline Bread and cereal (servings/week) & & $8.3 \pm 10.4$ & $0-98.0$ \\
\hline Beef (servings/week) & & $1.7 \pm 1.2$ & $0-5.9$ \\
\hline Butter and margarine (servings/week) & & $6.2 \pm 6.5$ & $0-49.0$ \\
\hline Alcohol (drinks/week) & & $2.4 \pm 4.7$ & $0-21.0$ \\
\hline \multicolumn{4}{|l|}{ C. Categorical variables } \\
\hline Race: Caucasian & $126(90.6 \%)$ & & \\
\hline African-American & $10(7.2 \%)$ & & \\
\hline Other & $3(2.2 \%)$ & & \\
\hline \multicolumn{4}{|l|}{ Menstrual status: } \\
\hline Premenopausal & $51(34.2 \%)$ & & \\
\hline Postmenopausal & $98(65.8 \%)$ & & \\
\hline \multicolumn{4}{|l|}{ Estrogen receptor status: } \\
\hline Positive & $80(73.4 \%)$ & & \\
\hline Negative & $29(26.6 \%)$ & & \\
\hline \multicolumn{4}{|l|}{ Lymph node status: } \\
\hline Negative & $85(57.0 \%)$ & & \\
\hline Positive & $64(43.0 \%)$ & & \\
\hline Stage: In situ & $29(19.6 \%)$ & & \\
\hline I & $51(34.5 \%)$ & & \\
\hline II & $51(34.5 \%)$ & & \\
\hline III & $13(8.8 \%)$ & & \\
\hline IV & $4(2.7 \%)$ & & \\
\hline $\begin{array}{l}\text { Recurrence-free survival, } \\
\text { five-year (Kaplan-Meier estimate): }\end{array}$ & $79 \%$ & & \\
\hline $\begin{array}{l}\text { Overall survival, } \\
\text { five-year (Kaplan-Meier estimate): }\end{array}$ & $84 \%$ & & \\
\hline
\end{tabular}


However, triceps skinfold thickness and arm muscle circumference were not correlated with each other.

Table 2 shows the risks of recurrence and death for the patient sample in relation to each of the study variables. Among non-dietary patient characteristics, risk of recurrence was increased for OC use, nodepositive status, and advanced stage. For OC use, the risk of recurrence increased 1.28-fold with a $95 \%$ confidence interval $(\mathrm{CI})=1.03,1.60$ for each three years of use. For node-positive (compared with nodenegative) status, there was a 2.36 -fold increased risk $(95 \% \mathrm{CI}=1.01,5.49)$. For the stage, the risk increased 2.22 times $(95 \% \mathrm{CI}=1.02,4.81)$ for each successive increase in level. Among predictors of overall survival, muscularity was protective. The hazard ratio (HR), analogous to the relative risk derived from a logistic regression model, was 0.27 for each additional $10 \mathrm{~cm}$ of arm muscle circumference $(95 \% \mathrm{CI}=$ $0.09,0.86)$. For each $1 \mathrm{~cm}$ of arm muscle circumference, the HR was $0.92(95 \% \mathrm{CI}=0.82,1.04)$ for recurrence and $0.88(95 \% \mathrm{CI}=0.79,0.99)$ for death. ER-negative status was associated with an increased risk of dying $(\mathrm{HR}=2.46 ; 95 \% \mathrm{CI}=1.02,5.94)$ compared with ER-positive status. Node-positive (vs. node-negative) status was a strong predictor of death $(\mathrm{HR}=3.31 ; 95 \% \mathrm{CI}=1.38,7.95)$. Finally, tumor stage was also strongly related to the risk of dying $(\mathrm{HR}=4.96 ; 95 \% \mathrm{CI}=1.86,13.23)$. Neither age nor menstrual status was found to be related to either outcome measure.

Dietary factors were examined both before and after adjusting for total energy. Total energy intake was itself associated with a 1.58-fold increased risk $(95 \% \mathrm{CI}=1.05,2.38)$ of recurrence for every additional $4,184 \mathrm{~kJ} /$ day $(1,000 \mathrm{kcal} /$ day $)$ in the diet. For every additional $418 \mathrm{~kJ} /$ day $(100 \mathrm{kcal} /$ day $)$ in the diet, the HR was $1.05(95 \% \mathrm{CI}=1.00,1.09)$ for recurrence. Closely related to the energy effect was that of total fat and fat subtypes, unadjusted for energy intake. For example, saturated fat intake was associated with a 1.79 -fold increase in the risk of recurrence for every additional $20 \mathrm{~g} /$ day in the diet $(95 \% \mathrm{CI}=1.05,3.04)$. Increased intakes of both saturated and polyunsaturated fats were also associated with increased risk of death. For polyunsaturated fat, this amounted to a 1.84-fold increased risk for each additional $10 \mathrm{~g} /$ day in the diet $(95 \% \mathrm{CI}=1.09,3.13)$. Total carotene and its principal food source, dark green and deep yellow vegetables, were associated with increased risks of recurrence. For carotene, this amounted to a 1.19-fold increased risk for each additional increment of $2,000 \mu \mathrm{g} /$ day in the diet $(95 \% \mathrm{CI}=1.06,1.34)$. However, neither carotene nor green/yellow vegetables showed any relationship with the risk of death. After adjusting for total energy intake, all of the above dietary relationships disappeared, and in the process, a protective effect of breads and cereals $(\mathrm{HR}=0.55 ; 95 \% \mathrm{CI}=0.33,0.93$ for each increment of seven weekly servings) on risk of recurrence was revealed.

Table 3 shows the stratum-specific risks, by menstrual status, of recurrence and death in relation to total energy as well as to the other dietary variables after energy-adjustment. The only statistically significant finding in this analysis was the protective effect of bread and cereal on recurrence in postmenopausal subjects $(\mathrm{HR}=0.32 ; 95 \% \mathrm{CI}=0.14,0.71)$. Table 4 shows the stratum-specific risks, this time by BMI, of recurrence and death in relation to total energy and energy-adjusted dietary variables. In subjects in whom BMI was $\leq 27.0 \mathrm{~kg} / \mathrm{m}^{2}$, energy intake was associated with a 1.55-fold increased risk of recurrence for each additional 4,184 kJ/day $(1,000 \mathrm{kcal} /$ day $)(95 \% \mathrm{CI}=$ $1.02,2.36$ ), or a 1.04-fold increased risk for each additional $418 \mathrm{~kJ} /$ day $(100 \mathrm{kcal} /$ day $)$ in the diet $(95 \% \mathrm{CI}=$ $1.00,1.09)$. Among those with BMI $>27.0 \mathrm{~kg} / \mathrm{m}^{2}$, monounsaturated fat intake (HR $=10.70$ for each additional $15 \mathrm{~g} /$ day of intake; $95 \% \mathrm{CI}=1.15,99.19$ ) was found to be a strong predictor of early recurrence. Once again, however, no variable was associated with increased risk of death in either stratum.

Stratum-specific risks of recurrence and death in relation to total energy and energy-adjusted dietary variables were also examined by tumor stage (data not shown). In subjects with early stage (in situ or Stage I) disease, the risk of recurrence was elevated in patients with higher intakes of carotene $(\mathrm{HR}=1.42 ; 95 \% \mathrm{CI}=1.11,1.81)$ or its principal food source, dark green and deep yellow vegetables $(\mathrm{HR}=1.46 ; 95 \% \mathrm{CI}=1.01,2.13)$. In those with later stage illness (Stages II-IV), monounsaturated fat $(\mathrm{HR}=6.87 ; 95 \% \mathrm{CI}=1.56,30.35)$ was associated with an increased risk of recurrence, while fiber $(\mathrm{HR}=0.29 ; 95 \% \mathrm{CI}=0.09,0.89$ for each $10 \mathrm{~g} /$ day increment) afforded protection. In subjects with later stage breast cancer, the risk of death was also elevated with increased total energy consumption ( $\mathrm{HR}=$ 1.57 for each additional 4,184 kJ/day (1,000 kcal/day); $95 \% \mathrm{CI}=1.07,2.30 ; \mathrm{HR}=1.05$ for each additional $418 \mathrm{~kJ} /$ day $(100 \mathrm{kcal} /$ day $) ; 95 \% \mathrm{CI}=1.01,1.09)$.

Finally, Table 5 shows the strongest dietary and overall multivariate models of recurrence and death in the population as a whole. First, for the endpoint of recurrence, a model comprised only of dietary terms showed an increased risk for energy (HR = 
Table 2. Cox regression of breast cancer recurrence and death as predicted by patient characteristics and dietary factors ${ }^{\mathrm{a}}$

\begin{tabular}{|c|c|c|c|c|}
\hline & \multicolumn{2}{|c|}{ Recurrence $(n=28)$} & \multicolumn{2}{|c|}{ Death $(n=26)$} \\
\hline & $\begin{array}{l}\text { Hazard } \\
\text { ratio }\end{array}$ & $\begin{array}{l}95 \% \text { confidence } \\
\text { interval }\end{array}$ & $\begin{array}{l}\text { Hazard } \\
\text { ratio }\end{array}$ & $\begin{array}{l}95 \% \text { confidence } \\
\text { interval }\end{array}$ \\
\hline \multicolumn{5}{|l|}{ A. Patient characteristics and level } \\
\hline Age (per 10 years) & 0.87 & $0.65,1.17$ & 1.24 & $0.93,1.65$ \\
\hline Menstrual status (pre- vs. postmenopausal) & 0.99 & $0.46,2.15$ & 2.18 & $0.82,5.80$ \\
\hline Age at menarche (per year) & 0.78 & $0.58,1.05$ & 0.93 & $0.73,1.20$ \\
\hline Age at menopause (per 5 years) & 1.02 & $0.75,1.39$ & 1.03 & $0.76,1.39$ \\
\hline Parity (per live birth) & 1.15 & $0.89,1.48$ & 1.16 & $0.88,1.53$ \\
\hline Age at first birth (per 5 years) & 0.85 & $0.60,1.20$ & 0.93 & $0.64,1.35$ \\
\hline Number of children breastfed (per child) & 0.50 & $0.05,5.01$ & 2.80 & $0.30,25.90$ \\
\hline Weight (per $10 \mathrm{~kg}$ ) & 0.85 & $0.66,1.08$ & 0.96 & $0.76,1.21$ \\
\hline Body mass index ( $\left.>27.0 \mathrm{vs} . \leq 27.0 \mathrm{~kg} / \mathrm{m}^{2}\right)$ & 0.62 & $0.27,1.41$ & 0.74 & $0.32,1.71$ \\
\hline Arm muscle circumference $($ per $10 \mathrm{~cm}$ ) & 0.44 & $0.14,1.45$ & 0.27 & $0.09,0.86$ \\
\hline Oral contraceptives (per 3 years of use) & 1.28 & $1.03,1.60$ & 1.10 & $0.84,1.45$ \\
\hline Hormone replacement (per year of use) & 0.98 & $0.35,2.73$ & 0.78 & $0.28,2.19$ \\
\hline Mammograms (per mammogram) & 1.06 & $0.97,1.15$ & 0.77 & $0.61,0.98$ \\
\hline Estrogen receptor status (negative vs. positive) & 1.45 & $0.55,3.81$ & 2.46 & $1.02,5.94$ \\
\hline Lymph node status (positive vs. negative) & 2.36 & $1.01,5.49$ & 3.31 & $1.38,7.95$ \\
\hline Stage $(0$, I vs. II-IV) & 2.22 & $1.02,4.81$ & 4.96 & $1.86,13.23$ \\
\hline \multicolumn{5}{|l|}{ B. Dietary variable and level (unadjusted for energy): } \\
\hline Energy $($ per $1000 \mathrm{kcal} / \mathrm{d})$ & 1.58 & $1.05,2.38$ & 1.33 & $0.86,2.04$ \\
\hline Total fat $($ per $30 \mathrm{~g} / \mathrm{d})$ & 1.46 & $1.05,2.01$ & 1.38 & $0.99,1.91$ \\
\hline Saturated fat (per $20 \mathrm{~g} / \mathrm{d})$ & 1.79 & $1.05,3.04$ & 1.72 & $1.00,2.96$ \\
\hline Monounsaturated fat (per $15 \mathrm{~g} / \mathrm{d}$ ) & 1.65 & $1.09,2.49$ & 1.50 & $0.98,2.27$ \\
\hline Polyunsaturated fat (per $10 \mathrm{~g} / \mathrm{d}$ ) & 1.66 & $0.98,2.80$ & 1.84 & 1.093 .13 \\
\hline Fiber $($ per $10 \mathrm{~g} / \mathrm{d}$ ) & 1.28 & $0.92,1.77$ & 1.17 & $0.83,1.66$ \\
\hline Vitamin C (per $100 \mathrm{mg} / \mathrm{d})$ & 1.31 & $0.99,1.74$ & 1.07 & $0.77,1.48$ \\
\hline Carotene $($ per $2000 \mu \mathrm{g} / \mathrm{d})$ & 1.19 & $1.06,1.34$ & 1.04 & $0.85,1.27$ \\
\hline Retinol (per $500 \mu \mathrm{g} / \mathrm{d}$ ) & 1.07 & $0.85,1.33$ & 0.98 & $0.74,1.30$ \\
\hline Fruit (per 14 servings/week) & 1.22 & $0.85,1.75$ & 1.21 & $0.86,1.72$ \\
\hline Green and yellow vegetables (per 3 servings/week) & 1.30 & $1.05,1.59$ & 1.00 & $0.74,1.37$ \\
\hline Bread and cereal (per 7 servings/week) & 0.73 & $0.46,1.15$ & 0.90 & $0.64,1.27$ \\
\hline Beef (per 1.5 servings/week) & 1.04 & $0.67,1.61$ & 1.14 & $0.73,1.80$ \\
\hline Butter and margarine (per 5 servings/week) & 1.04 & $0.81,1.35$ & 1.11 & $0.88,1.40$ \\
\hline Alcohol (per 2 drinks/week) & 0.71 & $0.32,1.57$ & 1.09 & $0.81,1.46$ \\
\hline \multicolumn{5}{|l|}{ C. Dietary variable and level (adjusted for energy): } \\
\hline Fat (per $10 \%$ of energy) & 1.42 & $0.82,2.46$ & 1.27 & $0.71,2.27$ \\
\hline Protein (per 5\% of energy) & 0.67 & $0.39,1.51$ & 0.71 & $0.34,1.47$ \\
\hline Carbohydrate (per $10 \%$ of energy) & 0.98 & $0.63,1.52$ & 1.03 & $0.64,1.65$ \\
\hline Total fat $($ per $30 \mathrm{~g} / \mathrm{d})$ & 1.85 & $0.71,4.78$ & 1.51 & $0.55,4.14$ \\
\hline Saturated fat (per $20 \mathrm{~g} / \mathrm{d}$ ) & 1.89 & $0.47,7.59$ & 1.45 & $0.32,6.62$ \\
\hline Monounsaturated fat (per $15 \mathrm{~g} / \mathrm{d}$ ) & 2.33 & $0.76,7.11$ & 1.77 & $0.53,5.87$ \\
\hline Polyunsaturated fat (per $10 \mathrm{~g} / \mathrm{d}$ ) & 1.84 & $0.70,4.84$ & 2.21 & $0.81,5.98$ \\
\hline Fiber (per $10 \mathrm{~g} / \mathrm{d}$ ) & 0.92 & $0.48,1.76$ & 1.06 & $0.53,2.11$ \\
\hline Vitamin C (per $100 \mathrm{mg} / \mathrm{d})$ & 1.03 & $0.69,1.54$ & 0.92 & $0.57,1.47$ \\
\hline Carotene $($ per $2000 \mu \mathrm{g} / \mathrm{d})$ & 0.91 & $0.69,1.19$ & 0.91 & $0.69,1.20$ \\
\hline Retinol (per $500 \mu \mathrm{g} / \mathrm{d}$ ) & 0.86 & $0.55,1.37$ & 0.84 & $0.51,1.39$ \\
\hline Fruit (per 14 servings/week) & 0.96 & $0.63,1.45$ & 1.06 & $0.69,1.63$ \\
\hline Green and yellow vegetables (per 3 servings/week) & 1.17 & $0.90,1.52$ & 0.97 & $0.70,1.35$ \\
\hline Bread and cereal (per 7 servings/week) & 0.55 & $0.33,0.93$ & 0.87 & $0.58,1.29$ \\
\hline Beef (per 1.5 servings/week) & 0.89 & $0.57,1.39$ & 0.95 & $0.62,1.48$ \\
\hline Butter and margarine (per 5 servings/week) & 1.04 & $0.81,1.34$ & 1.11 & $0.88,1.40$ \\
\hline Alcohol (per 2 drinks/week) & 0.82 & $0.54,1.25$ & 1.02 & $0.82,1.27$ \\
\hline
\end{tabular}

\footnotetext{
${ }^{a}$ Each row represents a pair of separate Cox regression models, one for recurrence and one for death.
} 
Table 3. Cox regression of energy-adjusted dietary predictors of breast cancer recurrence and death, stratified by menstrual status ${ }^{\mathrm{a}}$

\begin{tabular}{|c|c|c|c|c|}
\hline \multirow[t]{2}{*}{ Dietary factor and level } & \multicolumn{2}{|c|}{ Recurrence } & \multicolumn{2}{|l|}{ Death } \\
\hline & $\begin{array}{l}\text { Hazard } \\
\text { ratio }\end{array}$ & $\begin{array}{l}95 \% \text { confidence } \\
\text { interval }\end{array}$ & $\begin{array}{l}\text { Hazard } \\
\text { ratio }\end{array}$ & $\begin{array}{l}95 \% \text { confidence } \\
\text { interval }\end{array}$ \\
\hline A. Premenopausal: & \multicolumn{2}{|c|}{$(n=10 / 51)$} & \multicolumn{2}{|c|}{$(n=5 / 51)$} \\
\hline Energy (per $1000 \mathrm{kcal})$ & 1.82 & $0.59,5.65$ & 1.11 & $0.23,5.45$ \\
\hline Fat (per $10 \%$ of energy) & 1.00 & $0.38,2.62$ & 1.65 & $0.35,7.68$ \\
\hline Protein (per 5\% of energy) & 0.82 & $0.26,2.55$ & 0.56 & $0.11,2.88$ \\
\hline Carbohydrate (per $10 \%$ of energy) & 1.24 & $0.58,2.65$ & 1.18 & $0.39,3.60$ \\
\hline Total fat (per $30 \mathrm{~g} / \mathrm{d}$ ) & 1.00 & $0.19,5.34$ & 2.40 & $0.17,4.75$ \\
\hline Saturated fat (per $20 \mathrm{~g} / \mathrm{d}$ ) & 0.35 & $0.02,5.80$ & 1.27 & $0.03,60.62$ \\
\hline Monounsaturated fat (per $15 \mathrm{~g} / \mathrm{d}$ ) & 0.88 & $0.10,7.64$ & 3.94 & $0.12,127.87$ \\
\hline Polyunsaturated fat (per $10 \mathrm{~g} / \mathrm{d}$ ) & 2.68 & $0.65,11.10$ & 5.45 & $0.79,37.58$ \\
\hline Fiber (per $10 \mathrm{~g} / \mathrm{d}$ ) & 1.07 & $0.34,3.33$ & 0.42 & $0.06,3.04$ \\
\hline Vitamin C (per 100 mg/d) & 0.96 & $0.46,2.01$ & 0.49 & $0.11,2.14$ \\
\hline Carotene (per $2000 \mu \mathrm{g} / \mathrm{d}$ ) & 1.12 & $0.87,1.44$ & 0.56 & $0.14,2.21$ \\
\hline Retinol (per $500 \mu \mathrm{g} / \mathrm{d}$ ) & 1.32 & $0.41,4.25$ & 2.43 & $0.47,12.50$ \\
\hline Fruit (per 14 servings/week) & 0.56 & $0.21,1.49$ & 0.17 & $0.01,1.86$ \\
\hline $\begin{array}{l}\text { Green and yellow vegetables } \\
\text { (per } 3 \text { servings/week) }\end{array}$ & 1.38 & $0.94,2.03$ & 0.96 & $0.45,2.02$ \\
\hline Bread and cereal (per 7 servings/week) & 1.09 & $0.55,2.13$ & 1.19 & $0.48,2.94$ \\
\hline Beef (per 1.5 servings/week) & 0.57 & $0.20,1.59$ & 0.54 & $0.12,2.34$ \\
\hline Butter and margarine (per 5 servings/week) & 0.73 & $0.35,1.52$ & 0.73 & $0.35,1.52$ \\
\hline Alcohol (per 2 drinks/week) & 0.41 & $0.01,16.35$ & 0.41 & $0.01,16.35$ \\
\hline B. Postmenopausal: & \multicolumn{2}{|c|}{$(n=18 / 98)$} & \multicolumn{2}{|c|}{$(n=21 / 98)$} \\
\hline Energy (per $1000 \mathrm{kcal})$ & 1.56 & $0.99,2.46$ & 1.26 & $0.81,1.97$ \\
\hline Fat (per $10 \%$ of energy) & 1.05 & $0.98,1.13$ & 1.03 & $0.96,1.09$ \\
\hline Protein (per 5\% of energy) & 0.94 & $0.79,1.12$ & 0.93 & $0.79,1.10$ \\
\hline Carbohydrate (per $10 \%$ of energy) & 0.99 & $0.93,1.04$ & 1.00 & $0.95,1.05$ \\
\hline Total fat (per $30 \mathrm{~g} / \mathrm{d}$ ) & 2.46 & $0.79,7.66$ & 1.55 & $0.53,4.51$ \\
\hline Saturated fat (per $20 \mathrm{~g} / \mathrm{d}$ ) & 3.54 & $0.73,7.26$ & 1.77 & $0.36,8.65$ \\
\hline Monounsaturated fat (per $15 \mathrm{~g} / \mathrm{d}$ ) & 3.27 & $0.91,11.69$ & 1.63 & $0.47,5.67$ \\
\hline Polyunsaturated fat (per $10 \mathrm{~g} / \mathrm{d}$ ) & 1.36 & $0.36,5.15$ & 1.86 & $0.55,6.24$ \\
\hline Fiber (per $10 \mathrm{~g} / \mathrm{d}$ ) & 0.84 & $0.38,1.88$ & 1.14 & $0.55,2.36$ \\
\hline Vitamin C (per 100 mg/d) & 1.06 & $0.66,1.70$ & 0.97 & $0.59,1.59$ \\
\hline Carotene (per $2000 \mu \mathrm{g} / \mathrm{d})$ & 1.25 & $0.92,1.69$ & 1.12 & $0.80,1.58$ \\
\hline Retinol (per $500 \mu \mathrm{g} / \mathrm{d}$ ) & 0.78 & $0.45,1.36$ & 0.65 & $0.36,1.18$ \\
\hline Fruit (per 14 servings/week) & 1.16 & $0.73,1.84$ & 1.23 & $0.79,1.91$ \\
\hline $\begin{array}{l}\text { Green and yellow vegetables } \\
\text { (per } 3 \text { servings/week) }\end{array}$ & 1.04 & $0.72,1.50$ & 0.95 & $0.65,1.39$ \\
\hline Bread and cereal (per 7 servings/week) & 0.32 & $0.14,0.71$ & 0.76 & $0.48,1.22$ \\
\hline Beef (per 1.5 servings/week) & 1.11 & $0.63,1.93$ & 1.15 & $0.68,1.94$ \\
\hline Butter and margarine (per 5 servings/week) & 1.19 & $0.88,1.61$ & 1.11 & $0.82,1.50$ \\
\hline Alcohol (per 2 drinks/week) & 0.88 & $0.61,1.27$ & 1.07 & $0.88,1.30$ \\
\hline
\end{tabular}

${ }^{\mathrm{a}}$ Each row represents a pair of separate Cox regression models, one for recurrence and one for death. 
Table 4. Cox regression of energy-adjusted dietary predictors of breast cancer recurrence and death, stratified by body mass index ${ }^{\mathrm{a}}$

\begin{tabular}{|c|c|c|c|c|}
\hline \multirow[t]{2}{*}{ Dietary factor and level } & \multicolumn{2}{|c|}{ Recurrence } & \multicolumn{2}{|l|}{ Death } \\
\hline & $\begin{array}{l}\text { Hazard } \\
\text { ratio }\end{array}$ & $\begin{array}{l}\text { 95\% confidence } \\
\text { interval }\end{array}$ & $\begin{array}{l}\text { Hazard } \\
\text { ratio }\end{array}$ & $\begin{array}{l}95 \% \text { confidence } \\
\text { interval }\end{array}$ \\
\hline A. Body mass index $\leq 27.0 \mathrm{~kg} / \mathrm{m}^{2}$ & \multicolumn{2}{|c|}{$(n=20 / 92)$} & \multicolumn{2}{|c|}{$(n=18 / 92)$} \\
\hline Energy (per $1000 \mathrm{kcal}$ ) & 1.55 & $1.02,2.36$ & 1.43 & $0.93,2.17$ \\
\hline Fat (per $10 \%$ of energy) & 1.28 & $0.67,2.43$ & 1.57 & $0.75,3.28$ \\
\hline Protein (per 5\% of energy) & 0.76 & $0.36,1.59$ & 0.57 & $0.25,1.31$ \\
\hline Carbohydrate (per $10 \%$ of energy) & 1.05 & $0.64,1.73$ & 1.08 & $0.61,1.88$ \\
\hline Total fat (per $30 \mathrm{~g} / \mathrm{d}$ ) & 1.52 & $0.51,4.53$ & 2.15 & $0.61,7.54$ \\
\hline Saturated fat (per $20 \mathrm{~g} / \mathrm{d}$ ) & 1.55 & $0.28,8.62$ & 3.73 & $0.52,26.78$ \\
\hline Monounsaturated fat (per $15 \mathrm{~g} / \mathrm{d}$ ) & 1.52 & $0.42,5.55$ & 2.50 & $0.58,10.83$ \\
\hline Polyunsaturated fat (per $10 \mathrm{~g} / \mathrm{d}$ ) & 1.75 & $0.52,5.95$ & 2.60 & $0.72,9.36$ \\
\hline Fiber (per $10 \mathrm{~g} / \mathrm{d}$ ) & 1.23 & $0.56,2.70$ & 1.20 & $0.50,2.90$ \\
\hline Vitamin C (per 100 mg/d) & 1.19 & $0.77,1.82$ & 0.89 & $0.51,1.58$ \\
\hline Carotene (per $2000 \mu \mathrm{g} / \mathrm{d}$ ) & 1.23 & $0.99,1.51$ & 0.99 & $0.71,1.40$ \\
\hline Retinol (per $500 \mu \mathrm{g} / \mathrm{d}$ ) & 0.94 & $0.53,1.66$ & 0.82 & $0.41,1.65$ \\
\hline Fruit (per 14 servings/week) & 1.00 & $0.62,1.62$ & 1.03 & $0.60,1.75$ \\
\hline $\begin{array}{l}\text { Green and yellow vegetables } \\
\text { (per } 3 \text { servings/week) }\end{array}$ & 1.17 & $0.89,1.54$ & 0.94 & $0.65,1.37$ \\
\hline Bread and cereal (per 7 servings/week) & 0.70 & $0.38,1.17$ & 0.94 & 0.591 .49 \\
\hline Beef (per 1.5 servings/week) & 0.55 & $0.28,1.08$ & 0.79 & $0.43,1.44$ \\
\hline Butter and margarine (per 5 servings/week) & 1.05 & $0.75,1.45$ & 1.03 & $0.73,1.46$ \\
\hline Alcohol (per 2 drinks/week) & 0.90 & $0.64,1.28$ & 0.83 & $0.39,1.79$ \\
\hline B. Body mass index $>27.0 \mathrm{~kg} / \mathrm{m}^{2}$ & \multicolumn{2}{|c|}{$(n=8 / 57)$} & \multicolumn{2}{|c|}{$(n=8 / 57)$} \\
\hline Energy (per $1000 \mathrm{kcal})$ & 1.46 & $0.43,5.00$ & 0.71 & $0.22,2.29$ \\
\hline Fat (per $10 \%$ of energy) & 2.38 & $0.77,7.37$ & 0.95 & $0.35,2.59$ \\
\hline Protein (per 5\% of energy) & 0.73 & $0.16,3.27$ & 1.37 & $0.29,6.59$ \\
\hline Carbohydrate (per $10 \%$ of energy) & 0.75 & $0.30,1.88$ & 0.89 & $0.37,2.17$ \\
\hline Total fat (per $30 \mathrm{~g} / \mathrm{d}$ ) & 4.41 & $0.65,30.00$ & 0.92 & $0.17,5.06$ \\
\hline Saturated fat (per $20 \mathrm{~g} / \mathrm{d}$ ) & 4.43 & $0.42,46.40$ & 0.38 & $0.03,5.21$ \\
\hline Monounsaturated fat (per $15 \mathrm{~g} / \mathrm{d}$ ) & 10.70 & $1.15,99.19$ & 1.04 & $0.13,8.68$ \\
\hline Polyunsaturated fat (per $10 \mathrm{~g} / \mathrm{d}$ ) & 2.50 & $0.51,12.28$ & 2.10 & $0.41,10.70$ \\
\hline Fiber (per $10 \mathrm{~g} / \mathrm{d}$ ) & 0.52 & $0.14,2.00$ & 0.92 & $0.29,2.92$ \\
\hline Vitamin C (per 100 mg/d) & 0.66 & $0.26,1.68$ & 0.97 & $0.44,2.13$ \\
\hline Carotene (per $2000 \mu \mathrm{g} / \mathrm{d})$ & 1.02 & $0.65,1.61$ & 1.04 & $0.68,1.60$ \\
\hline Retinol (per $500 \mu \mathrm{g} / \mathrm{d}$ ) & 0.82 & $0.38,1.78$ & 0.91 & $0.43,1.89$ \\
\hline Fruit (per 14 servings/week) & 0.87 & $0.38,1.98$ & 1.12 & $0.54,2.34$ \\
\hline $\begin{array}{l}\text { Green and yellow vegetables } \\
\text { (per } 3 \text { servings/week) }\end{array}$ & 1.01 & $0.50,2.03$ & 1.03 & $0.51,2.05$ \\
\hline Bread and cereal (per 7 servings/week) & 0.29 & $0.08,1.05$ & 0.73 & $0.34,1.59$ \\
\hline Beef (per 1.5 servings/week) & 1.77 & $0.97,3.21$ & 1.36 & $0.67,2.75$ \\
\hline Butter and margarine (per 5 servings/week) & 1.15 & $0.65,2.05$ & 1.34 & $0.76,2.36$ \\
\hline Alcohol (per 2 drinks/week) & 0.35 & $0.02,8.00$ & 1.16 & $0.84,1.62$ \\
\hline
\end{tabular}

${ }^{\mathrm{a}}$ Each row represents a pair of separate Cox regression models, one for recurrence and one for death. 
Table 5. Strongest dietary and overall multivariate Cox regression models of breast cancer recurrence and death

\begin{tabular}{|c|c|c|c|}
\hline & $\begin{array}{l}\text { Significance } \\
(P \text { value })\end{array}$ & $\begin{array}{l}\text { Hazard } \\
\text { ratio }\end{array}$ & $\begin{array}{l}95 \% \text { confidence } \\
\text { interval }\end{array}$ \\
\hline \multicolumn{4}{|l|}{ Recurrence - dietary variables: } \\
\hline Energy (per 1,000 kcal) & 0.0236 & 1.64 & $1.07,2.53$ \\
\hline Breads and cereals (per 7 servings/week) ${ }^{\mathrm{a}}$ & 0.0266 & 0.56 & $0.33,0.93$ \\
\hline \multicolumn{4}{|l|}{ Recurrence - all variables: } \\
\hline Energy (per $1,000 \mathrm{kcal}$ ) & 0.0063 & 1.84 & $1.19,2.86$ \\
\hline Oral contraceptives (per 3 years of use) & 0.0143 & 1.34 & $1.06,1.69$ \\
\hline Tumor stage (per each stage increase) & 0.0508 & 1.48 & $1.00,2.20$ \\
\hline Breads and cereals (per 7 servings/week) ${ }^{\mathrm{a}}$ & 0.0728 & 0.63 & $0.38,1.04$ \\
\hline \multicolumn{4}{|l|}{ Death - dietary variables: } \\
\hline Polyunsaturated fat (per $10 \mathrm{~g} / \mathrm{d})^{\mathrm{b}}$ & 0.0234 & 1.84 & $1.09,3.13$ \\
\hline \multicolumn{4}{|l|}{ Death - all variables: } \\
\hline Tumor stage (per each stage increase) & 0.0000 & 2.88 & $1.82,4.57$ \\
\hline BMI $/ \mathrm{AMC}^{\mathrm{c}}\left(\right.$ per $\left.\mathrm{kg} / \mathrm{m}^{2} / \mathrm{cm}\right)$ & 0.0027 & 1.64 & $1.19,2.26$ \\
\hline Energy (per $1,000 \mathrm{kcal})$ & 0.0368 & 1.58 & $1.03,2.43$ \\
\hline
\end{tabular}

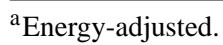

${ }^{\mathrm{b}}$ Non-energy-adjusted.

${ }^{\mathrm{c}} \mathrm{BMI}=$ body mass index, weight $(\mathrm{kg}) /$ height $\left(\mathrm{m}^{2}\right) ; \mathrm{AMC}=$ Arm muscle circumference $(\mathrm{cm})$.

$1.64 ; 95 \% \mathrm{CI}=1.07,2.53)$ and a decreased risk for bread and cereal $(\mathrm{HR}=0.56 ; 95 \% \mathrm{CI}=0.33,0.93)$. Total dietary fat and fat subtypes also contributed to significant predictive models in the absence of adjustment for total energy, but attenuated in effect or dropped out altogether from the multivariate models when the effect of energy was considered. When non-dietary variables were also introduced into multivariate models of recurrence, two variables, $\mathrm{OC}$ use $(\mathrm{HR}=1.34 ; 95 \% \mathrm{CI}=$ $1.06,1.69$ for every three years of use) and tumor stage $(\mathrm{HR}=1.48 ; 95 \% \mathrm{CI}=1.00,2.20$ for each increment in stage) were found to add to the predictive power and provide the most robust model.

In developing a model for predicting the outcome of death, the strongest dietary model consisted of only one statistically significant term: polyunsaturated fat intake unadjusted for energy intake $(\mathrm{HR}=1.84 ; 95 \% \mathrm{CI}=$ $1.09,3.13)$. Other fat subtypes, also unadjusted for energy, approached or achieved significance in bivariate models, but attenuated or dropped out of multivariate Cox regression models. When non-dietary patient characteristics were also introduced into a multivariate model of risk of death, energy (rather than fat or bread and cereal) intake was found to be the strongest dietary variable and to contribute to the most robust model. In addition to energy intake, two non-dietary variables were found to be very strong predictors of death: tumor stage and BMI adjusted for arm muscle circumference. Tumor stage was indeed a powerful predictor, with a 2.88 -fold increase $(95 \% \mathrm{CI}=1.82,4.57)$ in the risk of death for each one stage increase. BMI, on its own, failed to predict outcome in any setting. However, when adjusted for arm muscle circumference, BMI was a powerful predictor of risk of death with an attendant 1.64-fold increased risk $(95 \% \mathrm{CI}=1.19,2.26)$ for each incremental $\mathrm{kg} / \mathrm{m}^{2} / \mathrm{cm}$.

\section{Discussion}

We found several dietary and non-dietary factors to be associated with modification of risks of breast cancer recurrence and death following diagnosis. Higher levels of energy and fat intakes, OC use, ER-negative status, and advanced disease stage appear to be associated with an adverse effect on breast cancer outcome, whereas greater muscularity, increased intake of breads and cereals, and more frequent mammograms appear to be protective.

In the present study, prior to energy-adjustment, intakes of total fat, as well as of saturated and monounsaturated fat, were associated with increased risks of recurrence, and polyunsaturated fat intake was associated with increased risk of death. Among earlier studies 
that examined the relationship of diet with breast cancer outcome, several found an association between increased prediagnosis dietary fat intake and poorer survival [1-6], whereas others [7-9] failed to detect any relationship with fat intake. Other, but not all, studies found protective effects of intake of vegetables and fruits and their associated micronutrients, vitamin $C$ and $\beta$-carotene $[1,3,10]$. Several studies $[4,9,11-$ 15] have also implicated obesity in decreased survival, principally among postmenopausal patients.

Energy intake, however, may have accounted for at least part of the effect attributed to fat intake. A higher level of energy intake has been firmly established in animal models to be a potent mammary tumor promoter [29]. When compared with restricted energy intake, ad libitum intake has been shown to result in more tumors, more rapid onset, enhanced tumor growth, and more metastases [30]. It is conceivable that the tumorigenic effects of energy intake also pertain to humans, and therefore, it is not surprising that the adverse effects of fat were attenuated or disappeared upon energyadjustment. In fact, energy intake is closely related to intakes of many nutrients, particularly fat, and very high correlations (identical to those observed in this population) have been reported in numerous previous studies [30, 31]. In the present study, energy intake was found to be an important independent risk factor for both recurrence and death, and the effects of energy intake may even have been underestimated. Overweight women tend to under-report energy intake [32], thereby confounding energy intake effects with the possibly beneficial effects of leanness. Also, decreased reported energy intake may have resulted from advanced disease at diagnosis. Finally, in more physically fit subjects, increased energy intake could have been associated with a possibly beneficial increased expenditure. Therefore, the adverse effects of energy intake may have been spuriously underestimated.

Among other dietary relationships with outcome, intake of breads and cereals was found to be protective against recurrence in the present study. The beneficial effect was particularly pronounced in postmenopausal women $(\mathrm{HR}=0.32 ; 95 \% \mathrm{CI}=0.14,0.71)$. This protective effect may have occurred because these foods are a rich source of fiber, a non-nutritive dietary component which has been associated with lower circulating estradiol concentrations, increased fecal estrogen, and lower breast cancer incidence [33].

The unexpected association between carotene intake (when unadjusted for energy intake) and an increased risk of recurrence is difficult to interpret on the basis of results from previous studies. This associ- ation disappeared upon energy adjustment, reflecting carotene associations with intakes of energy and total dietary fat. Nevertheless, this finding was surprising because dietary (as opposed to supplemental) carotene has generally tended to be protective in terms of the incidence of breast and other cancers [34-36].

As expected, inherent tumor characteristics were most prognostically important among the non-dietary factors. Tumor stage was associated with recurrence and was the strongest of all predictors of death. Similarly, lymph node-positive status was also strongly predictive of both endpoints. ER-negative status was associated with death but was unrelated to disease recurrence. Mammographic utilization (frequency of mammograms during the five years prior to diagnosis), because of its ability to detect the disease at an earlier stage, was also associated with a decreased risk of death. Although associated with breast cancer incidence, reproductive factors (ages at menarche and menopause, age at first birth, gravida and parity, and breastfeeding) were unrelated to either endpoint.

Arm muscle circumference, a measure of lean body mass, was strongly associated with a decreased risk of death. This finding may have resulted from a beneficial effect of exercise and physical activity and is consistent with recent observations that breast cancer incidence is reduced in individuals who are more physically active [37-41]. However, it is also possible that it reflects a loss of lean muscle mass by patients with advanced disease. Nonetheless, the persistence of a protective effect of arm muscle circumference when controlling for tumor stage suggests that physical activity may indeed be beneficial. While no effects were noted for weight or BMI, the correction of BMI for muscularity yielded an index of adiposity that was directly associated with death. This was a stronger effect than that of arm muscle circumference in the opposite (protective) direction, and second in strength only to that of disease stage.

A number of mechanisms have been suggested to explain the relationship between excess energy intake, increased adiposity, and the promotion of mammary carcinogenesis, which has been observed in animal model systems $[29,30]$ and is suggested by the results of the present study. Most of these mechanisms relate to the hormonal and metabolic factors that are associated with increased energy intake or obesity. Insulin, secreted in response to meals to enable glucose clearance and energy metabolism, and insulin-like growth factors may have direct effect on the development and promotion of breast cancer [42]. Substantial evidence specifically implicates circulating estrogens as a key factor in modulating breast carcinogenesis [43-45], 
and dietary and physical activity patterns may influence risk for recurrence due to effects on estrogen production and metabolism. Adipose tissue provides the site for peripheral aromatization of androstenedione to estrone, a mechanism that may be particularly important in the promotion of postmenopausal breast cancer [46].

Finally, among the non-dietary factors, a history of OC use was strongly predictive of recurrence. The adverse effect of OC use was stronger in postmenopausal than in premenopausal women, a finding that is biologically feasible because postmenopausal subjects used OC in the distant past when the average estrogen concentration of these formulations was higher. No association between HRT and recurrence or death was observed.

Our study had several important limitations. The sample size may have limited the power to detect effects, particularly in stratified analyses. Similarly, the relative dietary homogeneity (with respect to fat and other nutrients) in this patient population may have attenuated risk estimates, biasing results toward the null. Therefore, even though significant relationships between intakes of energy, fat, and other dietary variables and outcome were observed, it is possible that a wider distribution of intakes or a greater sample size would have amplified effects which were underestimated or revealed those which were undetected. Also, only prediagnosis diet, reflecting the year prior to the examination and diagnosis, was assessed. It is possible that the subjects may have changed their diets after diagnosis, either as a consequence of their disease and its treatment or, possibly, in an attempt to reduce risk for recurrence or death. It is also possible that in those with advanced disease at the time of diagnosis, prediagnosis diet may have reflected taste or appetite changes resulting from the breast cancer. Notably, these women were not the target of dietary intervention efforts, and the likelihood of many spontaneous and substantial changes in well-established adult eating patterns seems small. Etiologic factors associated with risk of breast cancer are plausibly related to progression following the diagnosis of breast cancer [3], and additionally, prediagnosis dietary and activity patterns may even be among the determinants of tissue characteristics that influence prognosis [47].

Finally, the dietary data in the present study should be interpreted cautiously. FFQs are imprecise methods of dietary assessment. The potential bias toward the null, or an overestimate of intake, may have obscured relationships between the dietary variables and outcome factors. Data from FFQs may be particularly imprecise when micronutrient content data, such as carotenoids, are examined [48]. Use of the recently- released carotenoid database [25] in the dietary assessment would have enhanced the examination of dietary intakes of these compounds in this population. Nevertheless, total energy, fat, and other macronutrient intakes for this population were very close to values reported for women of a similar mean age from national surveys [49], providing confidence in the validity of the FFQ and the dietary data with respect to intakes of energy and macronutrients.

This study also had important strengths. First, recurrence was assessed as an outcome variable in addition to death. Most previous studies that have addressed the association between diet and breast cancer outcome have not examined the endpoint of disease recurrence [1, 3-6]. Although there is considerable overlap, risk factors for each of these two events were not identical. A second strength of this study is that nutrient estimates were energy-adjusted. Unfortunately, some of the earlier studies [4-6] failed to energy-adjust and were, therefore, unable to separate the effects of energy intake from those of other nutrient intakes. Finally, this study employed anthropometric assessments beyond weight, height, and BMI. This permitted a more refined analysis of the effects of weight and BMI by their relative adipose and muscle components [17], revealing relationships which would have otherwise been obscured.

In conclusion, we found that a combination of factors, including high intakes of energy and/or fat, obesity, and use of older formulations of OC may be associated with a poor prognosis after a diagnosis of breast cancer. Conversely, protection against breast cancer recurrence and death may be afforded by regular exercise (to promote increased lean body mass), a high intake of breads and cereals, and earlier detection through regular mammograms. Future research will aim to further refine these findings and to determine whether they are valid and abiding.

\section{Acknowledgements}

Supported by a research grant from Blue Cross - Blue Shield of Michigan. We thank the past and present members of the University of Michigan Breast Care Center who made this study possible, particularly Drs. Jay Harness, Allen Lichter, Lynne Carpenter, Hal Oberman, and David August. Special thanks also to Dr. Fred Wright for his valuable biostatistical guidance, to Dr. Jack McCue for his timely editorial assistance, and to Ms. Gretchen Miela for her help in obtaining Tumor Registry data. 


\section{References}

1. Jain M, Miller AB, To T: Premorbid diet and the prognosis of women with breast cancer. J Natl Cancer Inst 86: 13901397, 1994

2. Holm LE, Nordevang E, Hjalmar ML, Lidbrink E, Callmer E, Nilsson B: Treatment failure and dietary habits in women with breast cancer. J Natl Cancer Inst 85: 32-36, 1993

3. Rohan TE, Hiller JE, McMichael AJ: Dietary factors and survival from breast cancer. Nutr Cancer 20: 167-177, 1993

4. Zhang S, Folsom AR, Sellers IA, Kushi LH, Potter JD: Better breast cancer survival for postmenopausal women who are less overweight and eat less fat. The Iowa Women's Health Study. Cancer 76: 275-283, 1995

5. Nomura AMY, Le Marchand L, Kolonel LN, Hankin JH: The effect of dietary fat on breast cancer survival among Caucasian and Japanese women in Hawaii. Breast Cancer Res Treat 18(suppl): 135-141, 1991

6. Gregorio DI, Emrich LJ, Graham S, Marshall JR, Nemoto T: Dietary fat consumption and survival among women with breast cancer. J Natl Cancer Inst 75: 37-41, 1985

7. Kyogoku S, Hirohata T, Nomura Y, Shigematsu T, Takeshita S, Hirohata I: Diet and prognosis of breast cancer. Nutr Cancer 17: 271-277, 1992

8. Ewertz M, Gillanders S, Meyer L, Zedeler K: Survival of breast cancer patients in relation to factors which affect the risk of developing breast cancer. Int J Cancer 49: 526-530, 1991

9. Newman SC, Miller AB, Howe GR: A study of the effect of weight and dietary fat on breast cancer survival time. Am J Epidemiol 123: 767-774, 1986

10. Ingram D: Diet and subsequent survival in women with breast cancer. Br J Cancer 69: 592-595, 1994

11. Bastarrachea J, Hortobagyi GN, Smith TL, Kau SWC, Buzdar AU: Obesity as an adverse prognostic factor for patients receiving adjuvant chemotherapy for breast cancer. Ann Intern Med 119: 18-25, 1993

12. Senie RT, Rosen PP, Rhodes P, Lesser ML, Kinne DW: Obesity at diagnosis of breast cancer influences duration of disease-free survival. Ann Intern Med 116: 26-32, 1992

13. Boyd NF, Campbell JE, Germanson T, Thomson DB, Sutherland DJ, Meakin JW: Body weight and prognosis in breast cancer. J Natl Cancer Inst 67: 785-789, 1981

14. Tartter PI, Papatestas AE, Ioannovich J, Mulvihill MN, Lesnick G, Aufses AH: Cholesterol and obesity as prognostic factors in breast cancer. Cancer 47: 2222-2227, 1981

15. Donegan WL, Hartz AJ, Rimm AA: The association of body weight with recurrent cancer of the breast. Cancer 41: 15901594, 1978

16. Rock CL, Saxe GA, Ruffin MT, August DA, Schottenfeld D: Carotenoids, vitamin A, and estrogen receptor status in breast cancer. Nutr Cancer 25: 81-96, 1996

17. Heymsfield SB, Tighe A, Wang ZM: Nutritional assessment by anthropometric and biochemical methods. In: Shils M, Olson JA, Shike M (eds) Modern Nutrition in Health and Disease. Lea and Febiger, Philadelphia, 1994, pp 812-841

18. Willett W, Stampfer MJ: Total energy intake: implications for epidemiologic analyses. Am J Epidemiol 124: 17- 27, 1986

19. Kushi LH, Potter JD, Bostick RM, Drinkard CR, Sellers TA: Dietary fat and risk of breast cancer according to hormone receptor status. Cancer Epidemiol Biomarkers Prev 4: $11-19,1995$
20. Brown CC, Kipnis V, Freedman LS, Hartman AM, Schatzkin A: Energy-adjustment methods for nutritional epidemiology: the effect of categorization. Am J Epidemiol 139: 323-338, 1994

21. Beahrs O, Henson D, Hutter R, Myers M (eds): Manual for Staging of Cancer, 3rd edition. Lippincott, Philadelphia, 1988, pp 145-150

22. Block G, Hartman AM, Dresser CM, Carroll MD, Gannon J, Gardner L: A data-based approach to diet questionnaire design and testing. Am J Epidemiol 124: 453-469, 1986

23. Beecher GR, Chachik F: Evaluation of vitamin A and carotenoid data in food composition tables. J Natl Cancer Inst 73: 1397-1404, 1981

24. Mangels AR, Holden JM, Beecher GR, Forman MR, Lanza $\mathrm{E}$ : Carotenoid content of fruits and vegetables: an evaluation of analytic data. J Am Diet Assoc 93: 284-296, 1993

25. Chug-Ahuja JK, Holden JM, Forman MR, Mangels AR, Beecher GR, Lanza E: The development and application of a carotenoid database for fruits, vegetables, and selected multicomponent foods. J Am Diet Assoc 93: 318-323, 1993

26. Hankin JH, Le Marchand L, Kolonel LN, Wilkens LR: Assessment of carotenoid intakes in humans. Ann NY Acad Sci 691: 68-75, 1993

27. Cox DR: Regression models and life-tables. J R Stat Soc 34: 187-220, 1972

28. Manore M, Benardot D, Love P: Body measurements. In: Benardot D (ed) Sports Nutrition. American Dietetic Association, Chicago, 1992, pp 70-93

29. Klurfeld DM, Weber MM, Kritchevsky D: Inhibition of chemically-induced mammary and colon tumor promotion by calorie restriction in rats fed increased dietary fat. Cancer Res 47: 2759-2762, 1987

30. Albanes D: Energy intake and cancer. In: Micozzi MS, Moon TE (eds) Macronutrients: Investigating their Role in Cancer. Marcel Dekker, New York, 1992, pp 205-229

31. Willett W, Stampfer MJ: Total energy intake: implications for epidemiologic analyses. Am J Epidemiol 124: 17- 27, 1986

32. Lichtman SW, Pisarska K, Berman ER, Pestone M, Dowling H, Offenbacher E, Heshka S, Mathews DE, Heymsfield SB: Discrepancy between self-reported and actual caloric intake and exercise in obese subjects. N Eng J Med 327: 18931898, 1992

33. Lanza E, Shankar S, Trock B: Dietary fiber. In: Micozzi MS, Moon TE (eds) Macronutrients: Investigating their Role in Cancer. Marcel Dekker, New York, 1992, pp 293-319

34. Van Poppel G: Carotenoids and cancer: an update with emphasis on human intervention studies. Eur J Cancer 29A 1335- 1344, 1993

35. Comstock GGW, Bush TL, Helzlsouer K: Serum retinol, beta-carotene, vitamin E, and selenium as related to subsequent cancer of specific sites. Am J Epidemiol 135: 115-121, 1992

36. Potischman N, McCulloch CE, Byers T, Nemoto T, Stubbe $\mathrm{N}$ : Breast cancer and dietary and plasma concentrations of carotenoids and vitamin A. Am J Clin Nutr 52: 909-915, 1990

37. Thune I, Brenn T, Lund E, Gaard M: Physical activity and the risk of breast cancer. N Eng J Med 336: 1269-1275, 1997

38. Longnecker MP, Bernstein L, Paganini-Hill A, Enger SM, Ross RK: Risk factors for in situ breast cancer. Cancer Epidemiol Biomarkers Prev 5: 961-965, 1996 
39. D'Avanzo B, Nanni O, La Vecchia C, Franceschi S, Negri E, Giacosa A, Conti E, Montella M, Talamini R, Decarli A: Physical activity and breast cancer risk. Cancer Epidemiol Biomarkers Prev 5: 155-160, 1996

40. Bernstein L, Henderson BE, Hanisch R, Sullivan-Halley J, Ross RK: Physical exercise and reduced risk of breast cancer in young women. J Natl Cancer Inst 86: 1403-1408, 1994

41. Frisch RE, Wyshak G, Albright NL, Albright TE, Schiff I, Johns KP, Witski J, Shiang E, Koff E, Marguglio M: Lower prevalence of breast cancer and cancers of the reproductive system among former college athletes compared to nonathletes. Br J Cancer 52: 885-891, 1985

42. Westley BR, May FED: Insulin-like growth factors: the unrecognized oncogenes. Br J Cancer 72: 1065-1066, 1995

43. Key TJ, Pike MC: The role of oestrogens and progestagens in the epidemiology and prevention of breast cancer. Eur $\mathrm{J}$ Cancer Clin Oncol 24: 29-43, 1988

44. Pike MC, Spicer DV, Dahmoush T, Press MF: Estrogens, progestogens, normal breast cell proliferation and breast cancer risk. Epidemiol Rev 15: 17-35, 1993

45. Bernstein L, Ross RK: Endogenous hormones and breast cancer risk. Epidemiol Rev 15: 48-65, 1993
46. Le Marchand L: Anthropometry, body composition, and cancer. In: Micozzi MS, Moon TE (eds) Macronutrients: Investigating their Role in Cancer. Marcel Dekker, New York, 1992, pp 321-342

47. Russo J, Russo IH: Toward a physiologic approach to breast cancer prevention. Cancer Epidemiol Biomarkers Prev 3: 353-364, 1994

48. Briefel RR, Flegal KM, Winn DM, Loria CM, Johnson CL, Sempos CT: Assessing the nation's diet: limitations of the food frequency questionnaire. J Am Diet Assoc 92: 959962, 1992

49. Block G, Dresser CM, Hartman AM, Carroll MD: Nutrient sources in the American diet: quantitative date from the NHANES II Survey. II. Macronutrients and fats. Am J Epidemiol 122: 27-40, 1985

Address for offprints and correspondence: Cheryl L. Rock, $\mathrm{PhD}$, RD, Cancer Prevention and Control Program, University of California, San Diego, 9500 Gilman Drive, Dept. 0901, La Jolla, CA 92093-0901, USA; Tel: 619-822-1126; Fax: 619-822-1497 